\title{
FADO: A novel self-consistency spectral population synthesis tool for the exploration of galaxy evolution at high redshift
}

\author{
Jean Michel Gomes(i) \\ Instituto de Astrofísica e Ciências do Espaço, CAUP, Rua das Estrelas, \\ PT4150-762 Porto, Portugal \\ email: jeancastro.up.pt
}

\begin{abstract}
Despite significant progress over the past decades, all state-of-the-art population synthesis (PS) codes suffer from deficiencies limiting their potential of gaining sharp insights into the star formation history $(\mathrm{SFH})$ and Chemical Enrichment History $(\mathrm{CEH})$ of star-forming galaxies, i.e. the neglect of nebular continuum and, the lack of a mechanism to ensure consistency between the best-fitting SFH and the observed nebular characteristics (ONC; Balmer-lines, Balmer/ Paschen jumps). These introduce biases in their recovered physical properties (stellar mass $\mathrm{M}_{\star}$ and sSFR). FADO is a novel self-consistent PS code employing genetic optimization, publicly available (http:/ /www.spectralsynthesis.org), capable of identifying the SFH \& CEH that reproduce the ONC of a galaxy, alleviating degeneracies in the spectral fits. The current version of FADO (v1.b) uses standard BPT emission-line ratios for the classification of low redshift (z) galaxies. Whereas this permits a reliable distinction between star-forming, Composite, Seyfert and LINERs, it is inapplicable to many intermediate-z galaxies. We present an adaptation of FADO (version v1.c) to classify higher z galaxies employing the "Blue Diagram" (e.g., Lamareille $2010)$ for which the most prominent blue emission-lines $(<[\mathrm{OIII}] 5007 \AA)$ are observable while the $\mathrm{H} \alpha$ and $[\mathrm{NII}]$ are inaccessible. FADO v1.c was applied to synthetic spectra simulating the evolution of galaxies formed at higher-z with different SFHs. FADO can recover the physical and evolutionary properties of galaxies, such as $M_{\star}$ and mean age / metallicity, with an accuracy significantly better than purely-stellar codes. An outline of FADO v1.c and applications to local and intermediate-z galaxies will be presented.
\end{abstract}

Keywords. Spectral Synthesis, Stellar Populations, Nebular Emission, Galaxy Evolution, Galaxy Formation

\section{Introduction}

Spectral synthesis is one of the most fundamental and powerful tools available in modern extragalactic astronomy. It has been extensively used and improved over the past 50 years and has as its main goal to recover the mass-assembly history of galaxies from observed galaxy spectra. Two inverse yet complementary techniques have been developed so far: evolutionary and population synthesis, which are not seldom combined in one hybrid approach that involves, e.g., a search for the best-fitting model among a grid of synthetic spectral energy distributions (SEDs). The first one aims to shed light into galaxy evolution through comparison of observed galaxy SEDs with synthetic ones computed with simple assumptions on the star-formation and chemical enrichment history (SFH \& $\mathrm{CEH}$, respectively) of galaxies. The second approach, on the contrary, attempts to infer its elementary stellar building blocks (e.g., star clusters formed instantaneously with a given age and metallicity). 
Crucial and much-debated issues in spectral synthesis are the uniqueness of best-fitting SFHs \& CEHs solutions in the presence of the notorious age-metallicity-attenuation degeneracy, the requirements in signal-to-noise $(\mathrm{S} / \mathrm{N})$ for an accurate retrieval of the $\mathrm{SFH}$, and the possible modeling biases due to an incomplete description of physical ingredients (e.g., nebular emission) of the galaxy SED.

\section{Overview of the new version of FADO v1.c}

FADO stands for Fitting Analysis using Differential evolution Optimization (Gomes \& Papaderos 2017, 2018). This population synthesis code has two innovative concepts related to its mathematical and physical foundations. It is built using genetic differential evolution optimization and unsupervised clustering machine learning techniques to find the best-fit linear combination of stellar populations from galaxy spectra. Additionally, it incorporates both stellar \& nebular emission (continuum plus Balmer line-emission) in a self-consistent manner, i.e. FADO finds the best-match SFH that reproduces the nebular characteristics of a given galaxy, which alleviates known degeneracies that plague stellar population modelling (Cardoso, Gomes \& Papaderos 2019). So, FADO is well suited for the study of both young \& old stellar populations in galaxies.

Here we present the new upgraded version v1.c of FADO. There are several improvements related to the spectral fitting (Gomes et al., in prep.). We will briefly explain two main improvements related to intermediate and high-redshift galaxy spectral fitting.

FADO v1.b uses the standard BPT (Baldwin, Phillips \& Terlevich 1981) diagram for the spectral pre-classification of emission-line galaxies at low redshift in the optical. This has proven to be extremelly reliable to distinguish between star-forming, Composite, Seyfert 2 and LINERs. FADO v1.c implements a new classification scheme that uses the "Blue Diagram", i.e. for spectra redshifted out of the wavelength range of the red optical emission-lines ( $\mathrm{H} \alpha$ \& $[\mathrm{NII}]$ lines), but still containing the most prominent blue emissionlines in galaxies ([OIII] $5007 \AA, \mathrm{H} \beta,[\mathrm{OII}] 3727,3729 \AA)$. This calibration comes from Lamareille (2010) and minimizes the contamination from LINERs \& Seyfert 2 galaxies. It is defined as: $\log ([\mathrm{OIII}] 5007 / \mathrm{H} \beta)=0.11 /\{\log ([\mathrm{OII}] 3727,3729 / \mathrm{H} \beta)-0.92\}+0.85$

Therefore, from this work it was defined the galaxies that have a contamination from an active galactic nucleus: $\log ([\mathrm{OIII}] / \mathrm{H} \beta)>0.3$. The second important improvement in FADO is related to the pre-fitting of the underlying continuum in order to reliably estimate the emission-lines since the beginning of the fitting procedure. The new continuum determination uses a sliding box 'crap fitting'. This permits to have an idea of the nonsystematic errors of the spectrum and also to measure accuratelly emission-lines and equivalent widths, which in turn can be used with the self-consistent fitting in FADO from the very first run, helping towards convergence.

\section{Mock-galaxies: SED simulations test}

We have computed and subsequently modelled synthetic SEDs with the FADO population spectral synthesis code. The synthetic optical spectra were computed using distinct SFHs. For this, we adopted the Simple Stellar Population (SSP) models of the evolutionary synthesis code GALAXEV by Bruzual \& Charlot (2003), with Chabrier (2003) stellar initial mass function (IMF) between 0.1 and $100 \mathrm{M}_{\odot}$ and Padova evolutionary tracks (Alongi et al. 1993; Bressan et al. 1993; Fagotto et al. 1994a,b,c; Girardi et al. 1996).

The synthetic SEDs were then fitted from 2175 to $6000 \AA$ simulating observations of the VLT/MOONS spectral range in the low-resolution mode for galaxies at redshifts $\sim 2$. We have constructed the FADO base out of SSPs from the GALAXEV evolutionary synthesis code by Bruzual \& Charlot (2003). It contains 25 ages from 1 Myr to $~ 13.7$ Gyr and 4 metallicities $\left(\mathrm{Z}=0.2,0.4,1 \& 2.5 \mathrm{Z}_{\odot}\right)$. Note that these base elements have the 


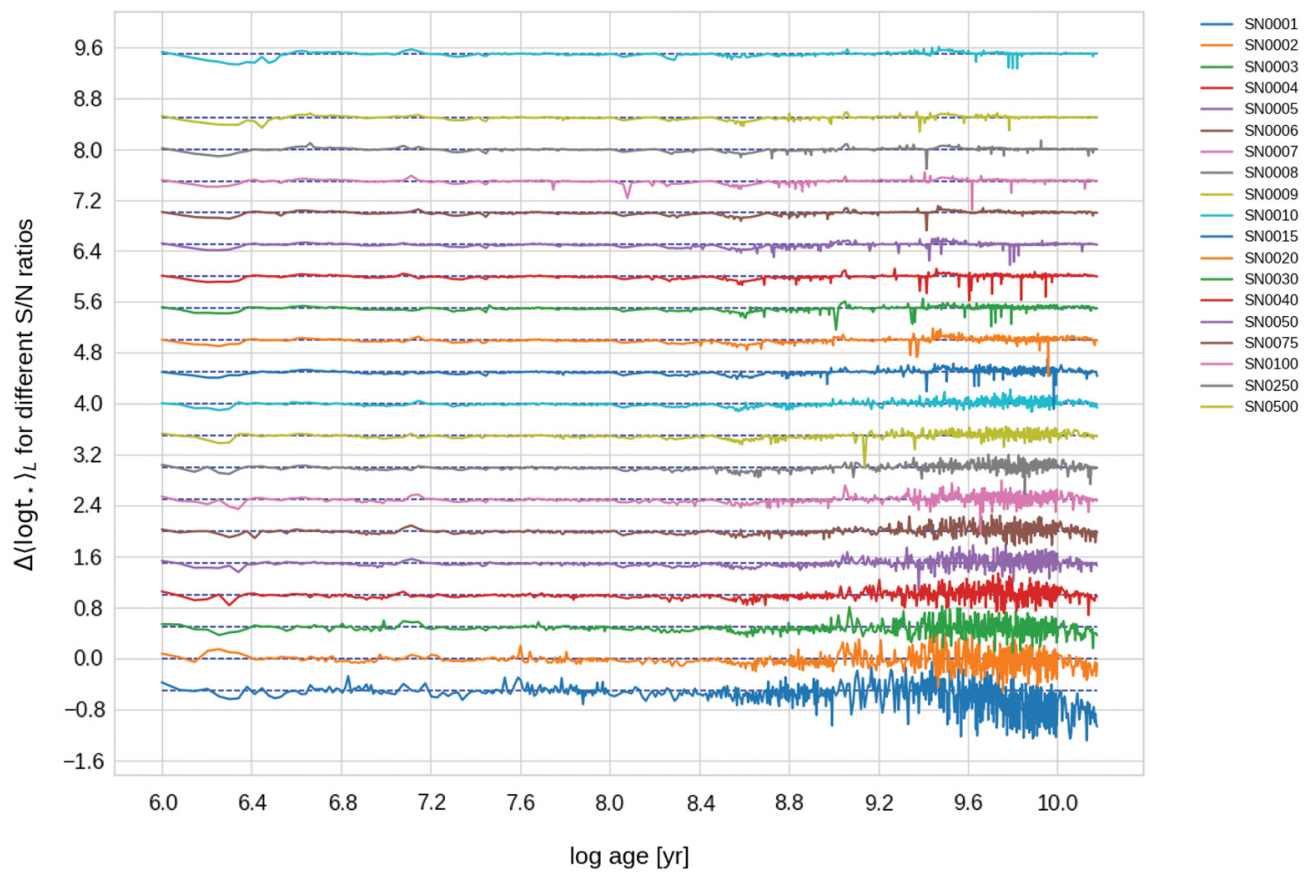

Figure 1. Difference between the output \& input luminosity-weighted mean stellar age $\left\langle\log t_{\star}\right\rangle_{L}$ as a function of the evolutionary age for an instantaneous burst model. Several S/N ratios at around $5020 \AA$ are shown increasing from bottom to top (see labels). The curves are shifted in the y-direction for better appreciation of the results. The spectral range in the FADO fits goes from 2175 to $6000 \AA$.

same ingredients as the generated mock-galaxy spectra. This is to try to map the intrinsic degeneracies of the fits. We show in figure 1 the difference (output-input) in the mean stellar age light-weighted as a function of the age for an instantaneous burst model (values similar to other SFHs tested, e.g., constant, exponentially declining) \& several $\mathrm{S} / \mathrm{N}$ ratios spanning values from 1 to 500 at around $5020 \AA$. FADO v1.c recovers the parameters within 0.2 dex on average, however, it may reach $\sim 0.6$ dex for old ages if the $\mathrm{S} / \mathrm{N}$ is low $(\sim 1)$. A thorough investigation will be presented in a future article (Gomes et al., in prep.).

\section{FADO applications to LEGA-C: stellar populations}

The Large Early Galaxy Astrophysics Census (van der Wel et al. 2016; LEGA-C) is an ESO Public Spectroscopic Survey available for the astronomical community that has obtained deep continuum galaxy spectra of several thousand galaxies from redshifts between 0.6 to 1 , which corresponds to ages of the universe 5.86 and $7.92 \mathrm{Gyr}$, respectively. The adopted cosmology uses: $\Omega_{\mathrm{M}}=0.3, \Omega_{\Lambda}=0.7$, and $H_{0}=72 \mathrm{~km} \mathrm{~s}^{-1} \mathrm{Mpc}^{-1}$ (Spergel et al. 2007).

The galaxy spectra were downloaded from the LEGA-C DR2 survey (Straatman et al. 2018). These spectra were extracted using a pipeline to correct for Galactic attenuation assuming a uniform dust screen model and the Cardelli, Clayton \& Mathis (1989) reddening curve. The dust IR emission map from our galaxy was used in the estimation of the attenuation. This map was taken from Schlegel, Finkbeiner \& Davis (1998) with the new recalibration from Schlafly \& Finkbeiner (2011). All spectra were corrected to restframe wavelengths and fluxes prior to spectral modelling. The luminosity distances were 


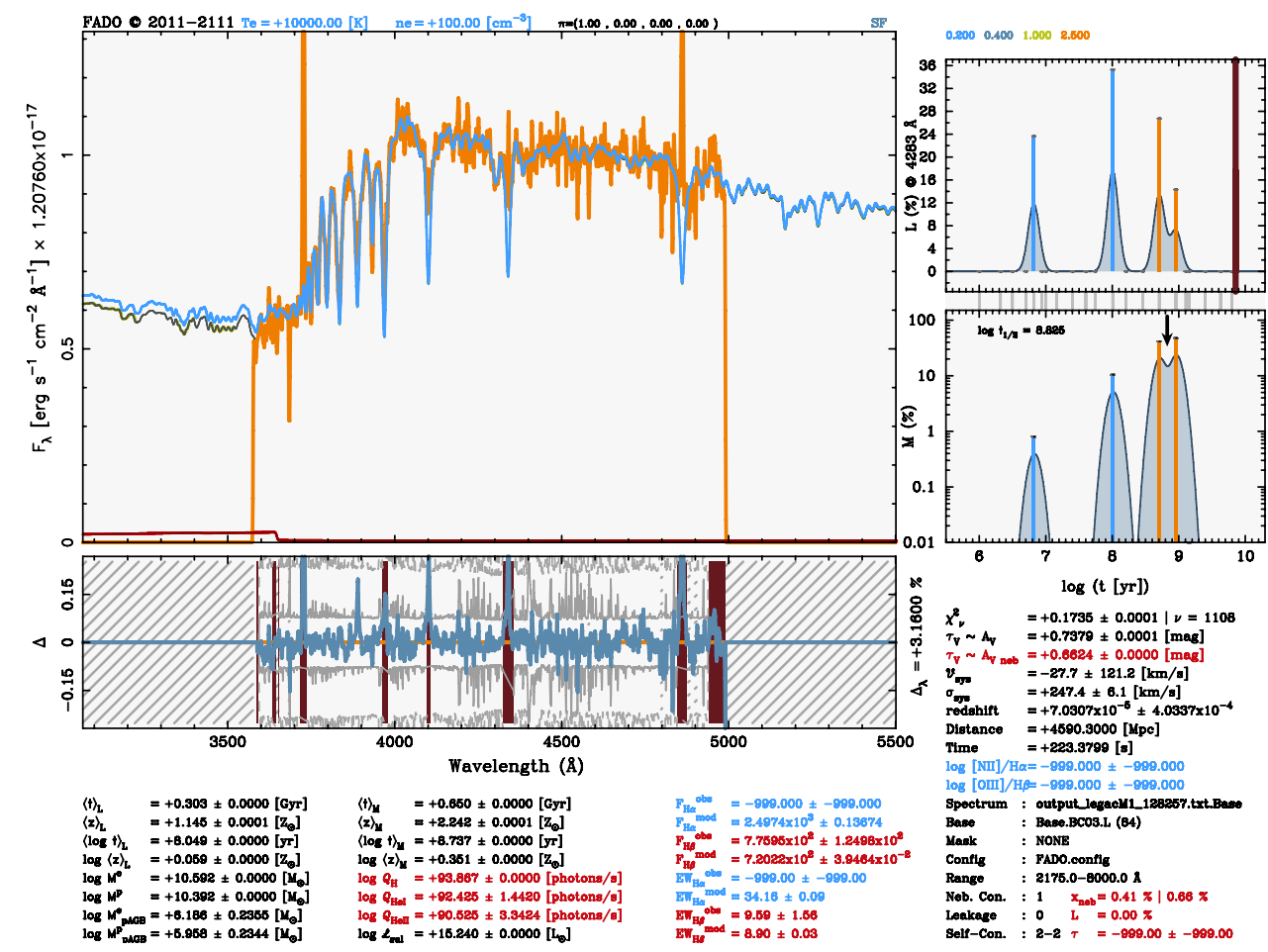

Figure 2. Example of a spectral fit with FADO of the star-forming galaxy M1 128257 from the LEGA-C survey. Top left panel: The LEGA-C spectrum of the source, corrected for Galactic attenuation (orange curve), reveals nebular emission in the blue region of the spectrum with a $\mathrm{H} \beta$ equivalent width of about $9.4 \AA$. Bottom left panel: residuals between fit and observed spectrum, with the shaded area and the dashed curve delineating, respectively, the $\pm 1 \sigma$ and $\pm 3 \sigma$ error spectrum. Right panels are the luminosity fraction (top) at the normalization wavelength (4283 $\AA$ ) and the stellar mass fraction (bottom) of the SSPs composing the best-fitting population vector as a function of their age. The color-coding depicts the metallicity and the vertical bars $\pm 1 \sigma$ uncertainties. The thin-gray vertical lines connecting both diagrams correspond to the ages of the SSPs in the used library. The light-blue shaded area in both panels shows smoothed version of the SFH using the Akima (1970) interpolation. The dark red line in the top right panel showing the light-fraction star formation history diagram depicts the maximum SSP age at the redshift 0.7269 of the source, i.e. the age of the universe at that redshift is $\sim 7.16 \mathrm{Gyr}$.

computed taking into account the infall onto Virgo supercluster, the Great Attractor, and the Shapley concentration using the methodology described in Mould et al. (2000). This was done to obtain absolute quantities from the spectral synthesis (e.g., stellar masses and luminosities).

To retrieve the star formation histories of our sample galaxies we used the population spectral synthesis code FADO v1.c. The spectra were not rebinned prior to the fitting since FADO has an automatic routine that flux-conserves the spectra. The FADO fits were performed using the full wavelength range of the LEGA-C spectra. The SSPs were the same as in Sect. 3. The upper bound on the age of the SSPs was chosen to be consistent with the maximum age of the universe, however, FADO automatically selects only stellar populations that are consistent with the redshift of the galaxy, i.e. the age of the universe at the redshift of observed galaxy. In our fits we have adopted the cosmology (Spergel et al. 2007). The SSPs encompass four metallicities: 0.2, 0.4, 1 \& $2.5 \mathrm{Z}_{\odot}$.

In Figure 2, we show an example of the spectral pixel-by-pixel modelling for galaxy M1 128257 of the LEGA-C survey, while in Figure 3 we show the mean stellar age versus 


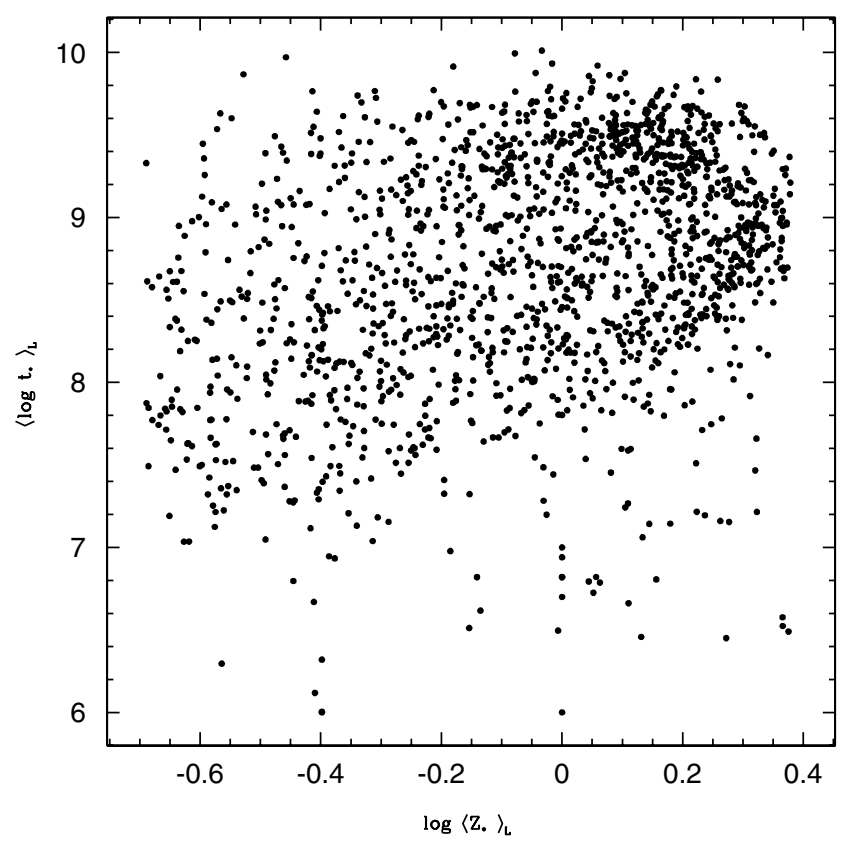

Figure 3. Mean stellar age versus mean stellar metallicity both luminosity-weighted for all LEGA-C galaxies where FADO has converged to a solution.

the mean stellar metallicity mass-weighted for all LEGA-C galaxies where FADO has converged. However, some of the galaxies are too noisy to be fitted.

\section{Acknowledgements}

J.M.G. acknowledges support by Fundação para a Ciência e a Tecnologia (FCT) through national funds (UID/FIS/04434/2013), FCT/MCTES through national funds (PIDDAC) by this grant UID/FIS/04434/2019 and by FEDER through COMPETE2020 (POCI-01-0145-FEDER007672). J.M.G. is supported by the DL 57/2016/CP1364/ CT0003 contract and acknowledges the previous support by the fellowships CIAAUP-04/ 2016-BPD in the context of the FCT project UID/FIS/04434/2013 \& POCI-01-0145FEDER-007672, and SFRH/BPD/66958/2009 funded by FCT and POPH/FSE (EC).

\section{References}

Akima, H. 1970, Journal of the ACM (JACM), 17, Issue 4, 589-602

Alongi, M., Bertelli, G., Bressan, A., et al. 1993, A\&AS, 97, 851

Bressan, A., Fagotto, F., Bertelli, G., \& Chiosi, C. 1993, A\& AS, 100, 647

van der Wel, A. et al. 2016, ApJS, 223, 29V

Baldwin, J. A., Phillips, M. M., \& Terlevich, R. 1981, PASP, 93, 5

Bruzual, G. \& Charlot, S. 2003, MNRAS, 344, 1000

Cardelli, J. A., Clayton, G. C., \& Mathis, J. S. 1989, ApJ, 345, 245

Chabrier, G. 2003, PASP, 115, 763

Cardoso, L. S. M., Gomes, J. M., \& Papaderos P. 2019, A\& A, 622, A56

Fagotto, F., Bressan A., Bertelli, G., \& Chiosi, C. 1994a, A $\& A S, 104,365$

Fagotto, F., Bressan, A., Bertelli, G., \& Chiosi C. 1994b, A\&AS, 105, 29

Fagotto, F., Bressan, A., Bertelli, G., \& Chiosi, C. 1994c, A\&AS, 105, 39

Girardi, L., Bressan, A., Chiosi, C., Bertelli, G., \& Nasi, E. 1996, A\&SAS, 117, 113

Gomes, J. M. \& Papaderos P. 2017, A\&A, 603, A63

Gomes, J. M. \& Papaderos, P. 2018, A\& A, 618, C3 
Lamareille, F. 2010, A\& A, 509, A53

Mould, J. R. et al. 2000, ApJ, 545, 547

Schlafly, E. F. \& Finkbeiner, D. P. 2011, ApJ, 737, 103

Schlegel, D. J., Finkbeiner, D. P., \& Davis, M. 1998, ApJ, 500, 525

Spergel, D. N. et al. 2007, ApJS, 170, 377

Straatman, C. M. S. et al. 2018, ApJS Series, 239, 27 